\title{
BRAGG GRATINGS PHOTOIMPRINTED IN INTEGRATED OPTICAL COMPONENTS: IMPROVING OF APODIZATION PROFILES
}

\author{
Lech Wosinski ${ }^{1}$, Romano Setzu ${ }^{2}$, and Matteo Dainese ${ }^{3}$ \\ Laboratory of Photonics and Microwave Engineering, Royal Institute of Technology (KTH), \\ Electrum 229, S-16440 Kista, Sweden \\ ${ }^{1}$ lech@imit.kth.se, ${ }^{2}$ itex_ros@it.kth.se.3matteo@imit.kth.se
}

\begin{abstract}
Apodized waveguide Bragg gratings are written using Sagnac type interferometer. As the quality of these gratings is not enough for DWDM devices, a procedure for shaping of filter profiles was developed. Obtained $10 \mathrm{~mm}$ long grating gives line width of $0.4 \mathrm{~nm}$ and almost completely suppressed side lobes.
\end{abstract}

\section{INTRODUCTION}

Silica-based planar lightwave circuits (PLCs) on a silicon platform will play a key role for fabrication of multifunctional devices for WDM network systems. Silica waveguides and waveguide devices will serve as communication paths between different elements on the platform as well as fill wavelength selective functions. Silica-on-silicon technology has shown the ability to keep high performance even for devices with high levels of integration, such as arrayed waveguide gratings.

Change of the refractive index of germanium doped silica under UV irradiation is commonly used for fabrication of UV imprinted fiber Bragg gratings. UVinduced changes of the refractive index in Ge-doped planar structures allow for adding new functionality to these components.

Imprinting of UV generated Bragg gratings in silica waveguides allows for building demultiplexers and add/drop filters [1] and can also be used for external cavity lasers to stabilize output wavelength [2]. UV processing of silica-based 
integrated devices also includes post-fabrication phase adjustment and wavelength tuning [3], direct UV-writing of waveguides [4], birefringence compensation by UV illumination [5] and UV-assisted poling [6]. WDM communication networks based on multifunctional integrated systems are a major step toward fully optical networking.

Bragg gratings with periodic modulation of the refractive index along the light guiding media were discovered in 1978 by Hill and co-workers in germanosilica optical fibers using illumination by an Ar-ion laser at $488 \mathrm{~nm}$ [7]. The use of UVlight at $244 \mathrm{~nm}$ was much more effective and the method proposed in 1989 by Meltz et al. [8] based on transverse holographic projection allowed to write gratings with any period by changing the angle between interfering beams. The experiment of Meltz and co-workers was a starting point for a dynamically grown research area of fiber Bragg gratings and later, waveguide gratings and related components.

Although it has gone more than 25 years since Hill's discovery, UV induced refractive index changes in germanosilicates are still not fully understood. Three main mechanisms that have been suggested by different research groups to explain the phenomena responsible for refractive index change in germanosilicate fiber cores due to UV illumination are: color-center model $[9,10]$, stress-relief or structural relaxation model $[11,12]$ and densification or compaction model [13, 14].

\section{MATERIAL ANALYSIS}

The photosensitivity of planar waveguides usually consists of contributions from the three mentioned processes and the net result is determined by their relative importance that depends on a number of factors.

A large compressive stress at the germanosilica - silicon interface plays here an important roll, but due to complicated microstructure the relaxation of this stress upon UV irradiation can be accompanied by localized compaction and positive index change.

Similarly to standard communication fibers the UV-induced refractive index change in germanosilicate films is usually not sufficient and hydrogen loading is necessary prior to UV-illumination. As hydrogen loading is not very convenient, especially in the case of planar components, increasing of photosensitivity by boron codoping has been used here as an interesting alternative. Boron doping has an opposite behavior on the refractive index, with respect to Ge. This means that B addition has a twofold advantage regarding UV photosensitivity, allowing higher Ge content for the same designed $\Delta$ as well as contributing itself to material densification upon UV exposure [15]. 
To test the boron influence on the UV photosensitivity of Ge doped silica films, three types of samples have been compared: a purely Ge doped PECVD deposited film as a reference, and two PECVD films with increasing boron content, 2 at\% and 5 at $\%$. The results are shown in Figure 1.

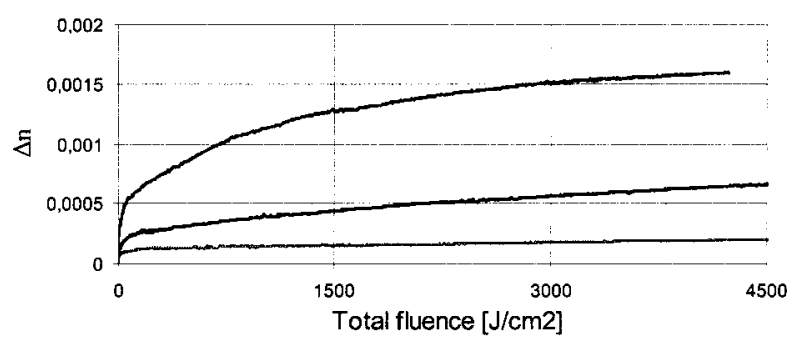

Figure 1. Variation of the refractive index in the germanium doped, boron codoped waveguide core upon UV exposure at $248 \mathrm{~nm}$. Pulse energy density $30 \mathrm{~mJ} / \mathrm{cm}^{2}$. Curves present from bottom: pure Ge-doped material, 2 at $\%$ B codoped, 5 at $\%$ B codoped.

The maximum obtained change of the refractive index due to UV illumination exceeded $1.5 \times 10^{-3}$, which is the result comparable to hydrogen loading. For further experiments we have chosen the film with higher boron codoping.

\section{EXPERIMENTAL SETUP}

For writing Bragg gratings in fibers a simple interferometrical method based on Sagnac interferometer is often used [16]. A similar system consisting of a continuous wave (CW) UV laser, an interferometer and a monitoring unit has been used here for writing gratings in waveguides (Figure 2).

A frequency doubled argon ion laser Coherent Innova 90 Fred provides a high quality laser beam at $244 \mathrm{~nm}$. The output power at this wavelength is $100 \mathrm{~mW}$ and the beam diameter is $0.6-0.8 \mathrm{~mm}$. A cylindrical lens system focuses the beam in the waveguide plane as a sharp $20 \mu \mathrm{m} \times 0.8 \mathrm{~mm}$ line. Such a formed beam is reflected from a motorized mirror $M M$ and then split mainly to the -1 and +1 diffraction orders at the phase mask. Zero- and higher-order diffracted beams have quite low efficiency and fall out of the system. The phase mask is used here as a beam splitter. The waveguide is placed just above the phase mask. The two beams go in opposite directions through mirrors M1 and M2 and then are recombined on the waveguide aside from the upcoming beams by turning the mirrors M1 and M2 in such a way that down-going beams are slightly tilted out of the interferometer plane. This tilt is very small and does not introduce visible distortions in the interference plane, when using the system for fibers.

By moving the motorized mirror MM along the phase mask, the interference pattern is moved along the waveguide allowing writing gratings with maximum 


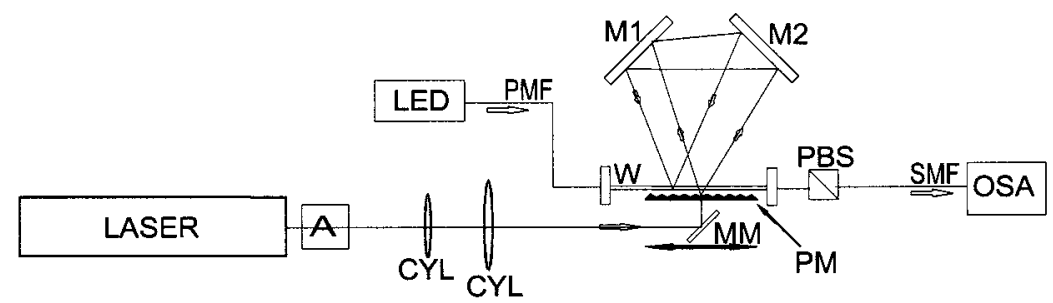

Figure 2. Setup for writing and evaluation of waveguide gratings with interferometrical method; LED - broadband light source, PMF - polarization maintaining fibre, SMF single mode fiber, PBS - polarisation beam splitter, OSA - optical spectrum analyser, A - programmable attenuator, CYL - cylindrical lens, MM - motorized mirror, $\mathrm{PM}$ - phase mask, M1, M2 -interferometer mirrors.

length equal to the length of the phase mask. The angle between the interfering beams at the waveguide plane determines the written grating period and depends on the phase mask period (diffraction angle) and tilt of mirrors M1 and M2. When the system is symmetric, the waveguide is situated at the same plane as the phase mask and the imprinting angle is equal the diffraction angle as in standard phase mask technique.

The spectral response of the grating is measured during the writing of the grating using a broadband light source and an optical spectrum analyzer.

The main drawback of this system in application to waveguides is the fact that for writing gratings in waveguides situated in larger distance from the sample edge, a considerable out of plane tilt of mirrors M1 and M2 must be introduced. The analytical study of the interferometer geometry and the subsequent computer simulations of the setup allowed to follow the beam path through the interferometer to the interference plane. To introduce minimum distortion the out of plane tilt of the interfering beams should be introduced by tilting of the mirror MM. This tilt causes that the interfering beams (focal lines) are crossing each other. The larger the tilt is, the crossing angle increases. This angle changes also when motorized mirror MM moves along the phase mask during grating writing. In result the illumination intensity as well as the length of the interference pattern responsible for writing gratings change also during the movement of the motorized mirror MM. As it will be shown later the gratings imprinted in waveguides suffer from the more significant side lobes on the shorter part of the spectrum in comparison to gratings imprinted in fibers.

\section{GRATING APODIZATION}

The spectral response of the grating is a Fourier transform of the envelope of the index modulation $\Delta n$ along the grating. In the simplest case, when a finite- 
length grating has a uniform modulation of refractive index, $\Delta n=$ const, as seen in Figure 3.a), the main peak or Bragg resonance in the spectral response is accompanied by a series of side lobes at adjacent wavelengths (sinc function). In WDM applications in which high rejection of adjacent channels is required it is important to lower the reflectivity of the side lobes, or to apodize the reflection spectrum of the grating.

A known method of suppressing the side lobes is to apodize the index profile such that towards the edges of the grating the index modulation approaches zero (Gaussian apodization) as seen in Figure 3.b). The gratings of this index profile show a significant suppression of side lobes, but on the short wavelength side residual peaks remain. Direct use of an expanded laser beam with a natural Gaussian intensity profile allows for the exposition of Gaussian apodized Bragg gratings.

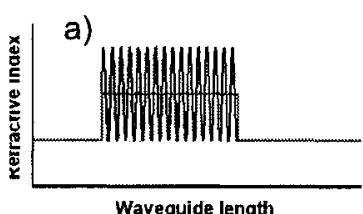

Waveguide length
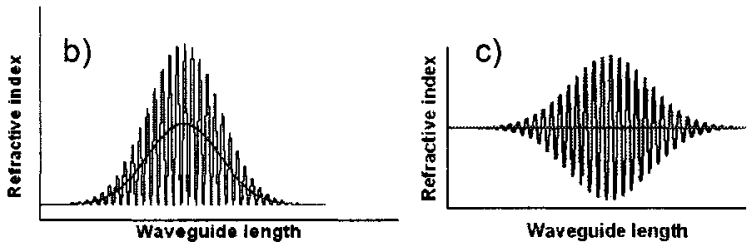

Figure 3. a): finite-length grating with uniform modulation of refractive index; b): self Gaussian apodization and c): raised Gaussian apodization of the laser intensity modulation for writing Bragg gratings. Line in the middle shows an average index profile. The period of the grating has been exaggerated for illustrative purposes.

Figure 4.a) and b) presents the simulated profile with Gaussian apodization and the spectral response of the grating obtained experimentally. Both profiles show the same behavior that can be explained by a non-uniform average index profile along the grating. Sections with a lower refractive index on both sides of the grating contribute to reflection of light with a shorter wavelength than the center part of the grating. Additionally these two reflections form Fabry-Perot resonances

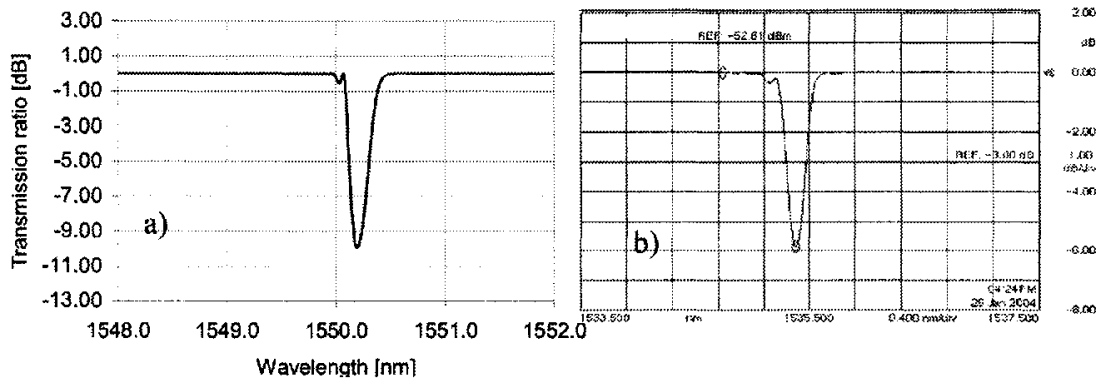

Figure 4. a) simulated profile of a grating with Gaussian apodization; b) transmission spectrum of a Bragg grating imprinted in a fiber with help of frequency doubled argon ion laser with natural Gaussian beam profile. 
that give additional peaks at the shorter wavelengths.

To suppress the side lobes caused by the described effect it is necessary to raise the average index of refraction to be constant along the grating length. An apodization using this index profile, the raised Gaussian apodization is shown in Figure 3.c) and the simulated filter profile as well as experimental transmission spectra of such a grating are shown in Figure 5.a) and b), respectively.

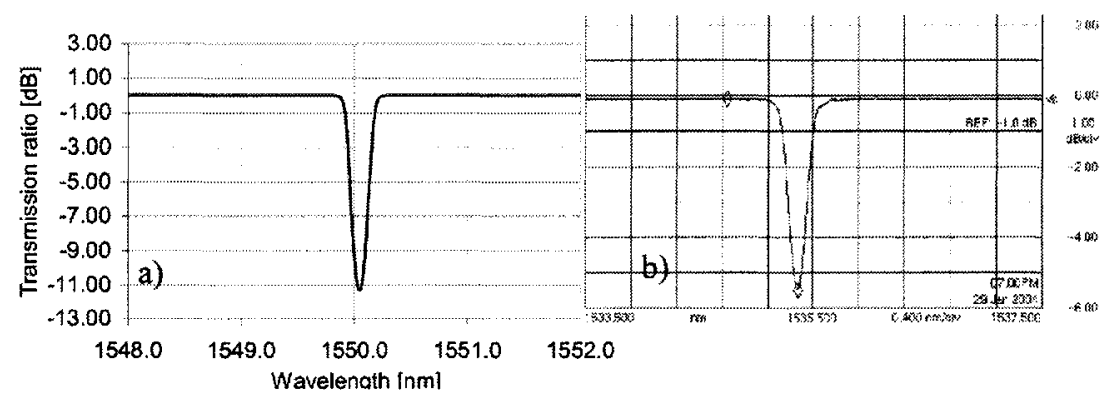

Figure 5. a) simulated profile of a grating with raised Gaussian apodization; b) transmission spectrum of a Bragg grating imprinted in a fiber with blanket post exposure.

To implement this apodization as well as other even more complicated apodization functions, programmable double exposure is necessary. Using a programmable tunable attenuator A and motorized mirror MM (shown in Figure 1) one can expose an arbitrarily chosen intensity profile along the grating to get an appropriate $\Delta n$ profile. Prior to this exposure or after it an "inverted" blanket exposure should be done, which gives an opposite profile of the mean refractive index change. Then, when overlapped with the modulated one a uniform average index profile along the grating is obtained.

In practice grating profiles usually obtained in planar waveguides contain additional deformations due to non-perfect alignment and out of plane distortions described earlier. Such filter profiles are not acceptable for the WDM applications as strong side lobes contribute to low separation between channels.

\section{FILTER PROFILE IMPROVEMENT}

A simple method that allows considerable improvement of the grating profile by choosing a suitable function for blanket post exposure has been developed.

A $10 \mathrm{~mm}$ long filter was exposed in the system shown in Figure 1 with Gaussian apodization profile with $10 \mathrm{~mm}$ length and FWHM 0.27. Three strong side lobes are clearly visible on the short wavelength side of the filter; one is of the same order of magnitude as the main peak (Figure 6.a)). To improve the shape of 
this filter, several post-exposures were performed over the same filter area. The five subsequent exposures have been done with inverted Gaussian profiles of different FWHM. The results are shown in Figure 6.b) - f).
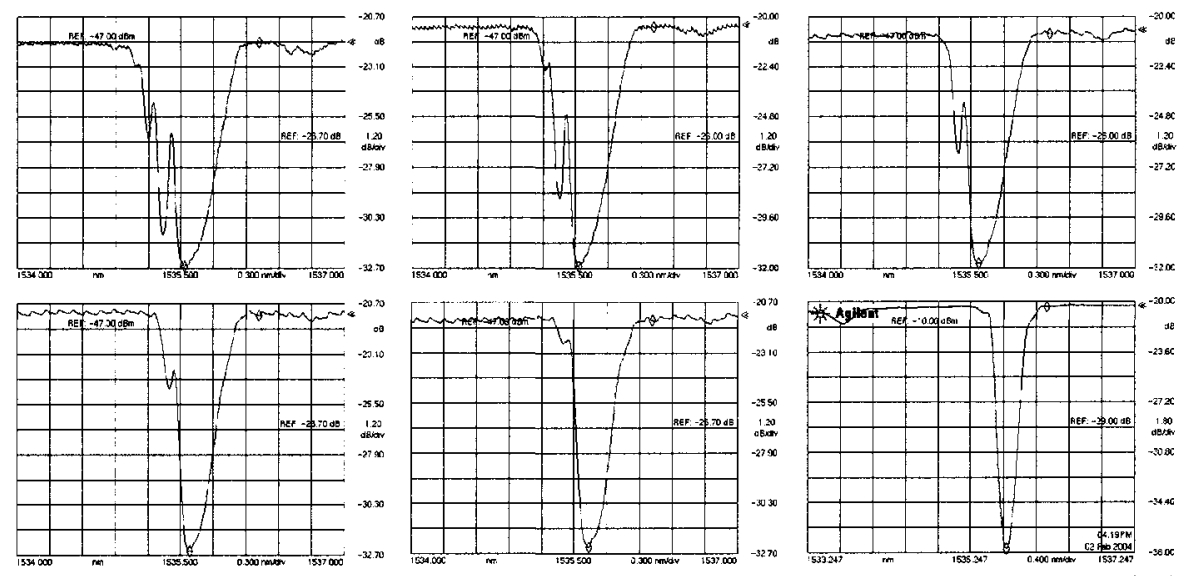

Figure 6. a) result after grating imprinted with Gaussian apodization profile with $10 \mathrm{~mm}$ length and FWHM $0.27 ; b)-f)$ : consecutive phases of apodization after blanket exposures with inverted Gaussian profile and FWHM: b) 0.27 , c) 0.21 , d) 0.21 , e) 0.162 and f) 0.162 .

Figure 6.f) shows the final result after post exposure completed. The raising of the refractive index at the edges of the grating by choosing suitable intensity profile has been geometrically adjusted to the side lobes characteristics. The side lobes on the shorter wavelength side have been almost completely erased, while the formation of longer wavelength side-lobes has been avoided. The improving process has been stopped when the right-side lobe started to be comparable with the left side one which means that the mean refractive index started to be higher on the edges than in the filter center. The obtained filter bandwidth (FWHM) decreased to $0.4 \mathrm{~nm}$ and grating strength reached $17.5 \mathrm{~dB}$, whereas side lobes observed $40 \mathrm{GHz}$ from the center of the filter decreased to the value below $0.5 \mathrm{~dB}$.

\section{CONCLUSIONS}

In this paper we have demonstrated a new technique for improving of apodization profiles of Bragg gratings imprinted in planar waveguides. This method is simple to implement and does not require any additional equipment beyond that for standard apodization. Suppressed side lobes below $0.5 \mathrm{~dB}$ for 17.5 $\mathrm{dB}$ grating have been demonstrated. Higher suppression of side lobes can be also achieved by changing the geometry of the interferometer to diminish out of plane distortions of the interferometric pattern. 


\section{REFERENCES}

[1] L. Wosinski, M. Dainese, H. Fernando and T. Augustsson, "Grating-assisted add-drop multiplexer realized in silica-on-silicon technology", Conference "Photonics Fabrication Europe", Brugge, Belgium, 28 October - 1 November 2002, Proc. SPIE 4941, 43 (2002).

[2] T. Tanaka, H. Takahashi, M. Oguma, T. Hashimoto, Y. Hibino, Y. Yamada, J. Albert, and K.O. Hill, "Integrated external cavity laser composed of spot-size converted LD and UV written grating in silica waveguide on $\mathrm{Si}^{\text {", }}$ Electron. Lett., 32, 1202-1203 (1996).

[3] M. Åslund, J. Canning and M. Bazylenko, "Tuning of integrated optical component using UV-induced negative index change", Electron. Lett., 35, 236-237 (1999).

[4] D.Zauner, K. Kulstad, J. Rathie and M. Svalgaard, "Directly UV-written silica-onsilicon planar waveguides with low insertion loss", Electron. Lett., 34, 1582-1584 (1998).J. Williams, "Narrow-band analyzer," Ph.D. dissertation, Dept. Elect. Eng., Harvard Univ., Cambridge, MA, 1993.

[5] J. Canning, M. Åslund, A. Ankiewicz, M. Dainese, H. Fernando, J. K. Sahu, and L. Wosinski, "Birefringence control in plasma-enhanced chemical vapor deposition planar waveguides by ultraviolet irradiation", Applied Optics, 39, 4296-4299 (2000).

[6] T. Fujiwara, D. Wong, Y. Zhao, S. Fleming, S. Poole, M. Sceats, "Electro-optic modulation in germanosilicate fibre with UV-excited poling", Electron. Lett. , 31, 573575 (1995).

[7] K. O. Hill, Y. Fujii, D. C. Johnson, and B. S. Kawasaki, "Photosensitivity in optical fiber waveguides: Application to reflection filter fabrication", Appl. Phys. Lett., 32, $647-649$ (1978).

[8] G. Meltz, W. W. Morey, and W. H. Glenn, "Formation of Bragg gratings in optical fibers by a transverse holographic method", Opt. Lett., 14, 823-825 (1989).

[9] D. L.Williams, S. T. Davey, R. Kashyap, J. R. Armitage, and B. J. Ainslie, "Direct observation of UV induced bleaching of $240 \mathrm{~nm}$ absorption band in photosensitive germanosilicate glass fibres," Electron. Lett. 28, 369-370 (1992).

[10] R. M. Atkins, V. Mizrahi, and T. Erdogan, " $248 \mathrm{~nm}$ induced vacuum UV spectral changes in optical fibre preform cores: support for a colour centre model of photosensitivity,' Electron. Lett. 29, 385-386 (1993).

[11] M. D. Sceats, S. B. Poole, "Stress-Relief - The Mechanism of Photorefractive Index Control in Fiber Cores", Proc. $16^{\text {th }}$ Australian Conference on Optical Fiber Technology", 302-305 (1991).

[12] M. D. Sceats, G. R. Atkins, and S. B. Poole, "Photolytic index changes in optical fibres," Ann. Rev. Mater. Sci. 23, 381-410 (1993).

[13] C. Fiori, R:A:B. Devine, "Ultraviolet irradiation induced compaction and photoetching in amorphous, thermal SiO2", Mat. Res. Soc., 61, 187-195 (1986).

[14] B. Poumellec, P. Guenot, I. Riant, P. Sansonetti, P. Niay, P. Bernage and J.F. Bayon, "UV induced densification during Bragg grating inscription in $\mathrm{Ge}: \mathrm{SiO} 2$ preforms", Opt. Mater., 4, 441-445 (1995).

[15] M. Douay, W. Xie, T. Taunay, P. Bernage, P. Niay, P. Cordier, B. Poumellec, L. Dong, J.F. Bayon, H. Poignant and E. Delevaque, "Densification involved in the UV-based photosensitivity of silica glasses and optical fibers", J. Lightwave Technol. 15, 8, 1329-1341 (1997).

[16] P.-Y. Cortés, H. Fathallah, S. laRochelle, L. A. Rusch and P. Loiselle, "Writing of Bragg gratings with wavelength flexibility using a Sagnac type interferometer and application to FH-CDMA", ECOC'98, 411 (1998). 